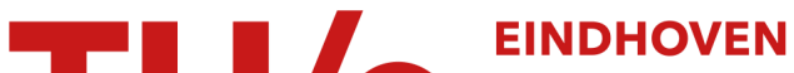 \\ UNIVERSITY OF \\ TECHNOLOGY
}

\section{Computational techniques for antenna engineering}

Citation for published version (APA):

Tijhuis, A. G., Beurden, van, M. C., \& Hon, de, B. P. (2008). Computational techniques for antenna engineering. In Microwave Wave Conference 2008, EuMC 2008 38th European Conference, London, UK, 27-31 Oct. 2008

(pp. 821-824). Institute of Electrical and Electronics Engineers. https://doi.org/10.1109/EUMC.2008.4751579

DOI:

10.1109/EUMC.2008.4751579

Document status and date:

Published: 01/01/2008

\section{Document Version:}

Publisher's PDF, also known as Version of Record (includes final page, issue and volume numbers)

\section{Please check the document version of this publication:}

- A submitted manuscript is the version of the article upon submission and before peer-review. There can be important differences between the submitted version and the official published version of record. People interested in the research are advised to contact the author for the final version of the publication, or visit the $\mathrm{DOI}$ to the publisher's website.

- The final author version and the galley proof are versions of the publication after peer review.

- The final published version features the final layout of the paper including the volume, issue and page numbers.

Link to publication

\section{General rights}

Copyright and moral rights for the publications made accessible in the public portal are retained by the authors and/or other copyright owners and it is a condition of accessing publications that users recognise and abide by the legal requirements associated with these rights.

- Users may download and print one copy of any publication from the public portal for the purpose of private study or research.

- You may not further distribute the material or use it for any profit-making activity or commercial gain

- You may freely distribute the URL identifying the publication in the public portal.

If the publication is distributed under the terms of Article 25fa of the Dutch Copyright Act, indicated by the "Taverne" license above, please follow below link for the End User Agreement:

www.tue.nl/taverne

Take down policy

If you believe that this document breaches copyright please contact us at:

openaccess@tue.nl

providing details and we will investigate your claim. 


\title{
Computational Techniques for Antenna Engineering
}

\author{
Anton G. Tijhuis ${ }^{1}$, Martijn C. Van Beurden ${ }^{2}$, Bastiaan P. de Hon ${ }^{3}$ \\ Department of Electrical Engineering, Eindhoven University of Technology \\ P.O. Box 513, $5600 \mathrm{MB}$ Eindhoven, the Netherlands \\ ${ }^{1}$ A.G.Tijhuis@tue.nl \\ ${ }^{2}$ M.C.v.Beurden@tue.nl \\ ${ }^{3}$ B.P.d.Hon@tue.nl
}

\begin{abstract}
In this paper, a two-stage approach is proposed for using computational electromagnetics in antenna engineering. First, stochastic optimization techniques are used in combination with approximate models. Second, line-search techniques are combined with full-wave modeling. The second stage is considered in detail; both the acceleration of the underlying field computations and the implementation of the optimization are discussed.
\end{abstract}

\section{INTRODUCTION}

The increasing complexity and high speed of devices in electrical engineering makes the wave character of the underlying physical phenomena increasingly more important. In telecommunications, radar as well as astronomy, there is a common need for integrated antennas. In addition, such antennas must be used for multiple functions. In RF structures, particularly the response of the interconnects requires the evaluation of electromagnetic fields in three dimensions. In EMC problems, the local behavior of a wiring network may affect the entire system. In all of these examples, the proximity of parts of the structure is such that probing the field causes a significant disturbance. Further, only a limited number of "observables" is extracted from the three-dimensional field.

This raises the question how computational electromagnetics can be used to analyze or even design such structures. In all cases, the complexity and the size of the device prevent a direct application of brute-force computational techniques. On the other hand, parts of such a geometry can be analyzed with state-of-the-art algorithms. In this paper, we elaborate on this situation and describe elements of an approach towards handling the desired complexity. The paper is intended to keynote a focused session; therefore, we present the general approach and stress those aspects that will not be addressed by other speakers. In particular, we describe the acceleration of the required forward modeling and the implementation of the sensitivity analysis with respect to the design parameters.

\section{Towards Electromagnetic EngineEring}

In the industrial design of a microwave or antenna system, typically two steps are followed. In the first stage, an extracted or equivalent model is optimized using one of the many available computational optimization strategies. In the second stage, a prototype of the resulting initial design is realized in hardware, and fine-tuned with the aid of experimental techniques. In both stages, human intelligence is needed to steer the process, in particular with respect to the choice of the design criteria and the choice of the direction in which the improvement or "update" is sought. This intelligence is usually obtained from a few experienced engineers, which makes the design process vulnerable and expensive. This raises the question whether computational techniques can be used to augment or even replace this process.

Engineering Electromagnetics has reached the stage where commercial software seems capable of replacing some or all of the prototyping in the second stage. However, a single electromagnetic-field computation for a complicated threedimensional configuration that is representative of an actual microwave or antenna device may still take hours or days. Thus, like in prototyping, only a few geometries are analyzed before the design is finalized. Stochastic techniques like genetic or particle-swarm optimization are capable of searching an optimum in a large parameter space with multiple local optima. However, they could have to analyze the electromagnetic behavior of many thousands of "candidates". Using state-ofthe-art computational electromagnetics for this purpose does not seem realistic for the foreseeable future.

This indicates that Electromagnetic Engineering, which aims at using computational electromagnetics in the synthesis problem, should follow a two-step strategy similar to the current industrial process. In the first stage, stochastic optimization is applied to approximate models that allow a fast evaluation of the cost or fitness function. Equivalent circuits or extracted parameters as they are widely used in engineering practice may be suitable for this purpose. An alternative approach is reduced-order modeling [1], [2], which is capable of reducing the size of a linear system of equations for an unknown field quantity to a much smaller system in which the behavior of relevant physical parameters is preserved.

In the second stage, we assume that a suitable initial estimate is available, either from the first stage or from engineering experience. Thus, local optima are avoided, and deterministic algorithms based on a local linearization may be employed for the optimization. Even this requires the evaluation of the electromagnetic behavior of tens of candidates, so that the computation time must still be reduced "from hours to minutes" for this procedure to be realistic.

Detection and synthesis problems have a lot in common. In both cases, the field in a configuration must be matched to specifications by a combination of modeling and optimization. 
For the simulations, this means that we must evaluate the field in a known geometry with a varying physical or line-search parameter. The difference lies in the formulation of the cost function for the optimization. For a detection problem, this involves a weighted least-squares summation over the deviation between the observed and the simulated field. In addition, the existence of an object is obvious, while its identity, shape and/or constitution must be determined uniquely. In a synthesis problem, the existence of a solution for a given specification is not a priori clear, while its uniqueness is less important. In the synthesis problem, therefore, the proper formulation of the cost function is a major challenge.

Last but not least, a device should be fault-tolerant. First, the design must account for variations due to the manufacturing. As an example, we mention the "profile dip" in optical fibers, which may show up in the fabrication of the preform from which the fiber is drawn. The influence of such variations can be determined from the sensitivities that are used to determine the search directions in line-search optimization. Second, the device may have to function in a varying environment. Here, the antenna in a mobile telephone may be a good example. The effect of such an environment can only be analyzed with the aid of a stochastic analysis.

\section{FORWARD MODELING}

In the remainder of this paper, we concentrate on the second stage as described in Section II, where an initial estimate is available. In that stage, full-wave modeling must be combined with line-search optimization, which amounts to successive sweeps with respect to a line-search parameter. Two concepts have been developed by our team to reduce the duration of a single field computation "from hours to minutes", so that it may be repeated tens of times in the optimization.

\section{A. Marching on in Anything}

The first concept concerns the solution of field problems for a varying physical parameter [3]. The parameter may be frequency, angle of incidence, object dimension, or a combination of physical quantities combined in a line-search parameter in an optimization step. After discretization, the field problem assumes the form of a linear system of equations

$$
L(p) u(p)=f(p),
$$

where $u(p)$ is a discretized field and $f(p)$ corresponds to the excitation. We are interested in the situation where this problem must be solved for a large number of sampled values of the parameter $p$, e.g., $p_{m}=p_{0}+m \Delta p$, with $m=$ $0,1, \ldots, M$. To this end, we minimize the squared error

$$
\operatorname{ERR}^{(n)}=\left\|r^{(n)}\right\|^{2}=<r^{(n)} \mid r^{(n)}>,
$$

with $r^{(n)}=L u^{(n)}-f$ with the aid of a standard conjugate gradient method. This procedure is accelerated significantly when the initial estimate for $p=p_{m}$ is generated from a few previous "final" results, according to

$$
u^{(0)}\left(p_{m}\right)=\sum_{k=1}^{K} \gamma_{k} u\left(p_{m-k}\right),
$$

where the $\left\{\gamma_{k} \mid k=1, \ldots, K\right\}$ are found by minimizing the squared error (2). The value of the coefficients $\left\{\gamma_{k}\right\}$ can be found from the system of linear equations

$$
\begin{array}{r}
\sum_{k=1}^{K}<L\left(p_{m}\right) u\left(p_{m-\ell}\right) \mid L\left(p_{m}\right) u\left(p_{m-k}\right)>\gamma_{k} \\
=<L\left(p_{m}\right) u\left(p_{m-\ell}\right) \mid f\left(p_{m}\right)>,
\end{array}
$$

with $\ell=1, \ldots, K$. The procedure has been demonstrated for boundary and domain integral equations in two and three dimensions. Typically $K=2$ or $K=3$, i.e., storing two or three previous final results suffices, and the acceleration rate varies between 10 and 50 .

For "finite" (difference or element) methods, the solution of the discretized equation (1) as such is less efficient because of the poor convergence of the conjugate gradient method. This can be explained from the structure of the adjoint operator which is employed to generate the update directions. This operator now links a few local field values, so that the update remains local as well. This problem can be remedied by applying a preconditioner based on a spectral decomposition of the discretized field. This opens up the possibility to combine the resulting preconditioned scheme with the extrapolation procedure outlined above.

\section{B. Diakoptics}

The second idea is to separate a large, complicated configuration into smaller subdomains. The electromagnetic field in these subdomains is then computed locally, for a simpler environment. Subsequently, the thus obtained field distributions are used as basis functions in a global version of the method of moments. This may be regarded as an application of Gabriel Kron's concept of diakoptics in electromagnetic field analysis.

This approach is increasingly used in modeling large, finite antenna arrays consisting of metallic patches. The currents on these patches are determined for an isolated patch and/or for a patch in an infinite array. In the synthetic functions (SFX, [4]) and characteristic basis function (CBF, [5]) approaches, this is achieved by moving an elementary source in the vicinity of the patch, and using the singular-value decomposition to extract independent distributions. In the eigenfunction approach [6], the eigencurrents of the integral operator for the isolated patch are used as basis functions. Thus, the choice of the varying excitation and the subsequent SVD are avoided. These methods have already been applied successfully to structures consisting of electrically isolated domains, while overlapping basis functions for electrically connected domains are emerging [4]. In all cases, the metallic surface on which the induced electric surface currents are computed is subdivided.

An alternative is to subdivide the entire three-dimensional space. Typically, we consider an observation domain and an environment. The environment is supposed to be fixed, while the constitution of the observation domain is varied and optimized. Again, different possibilities exist to realize this concept. For closed or fully periodic structures a decomposition into modes has led to the multimode equivalent 
network approach [7]. In recent years, this method has been extended from waveguiding structures to periodic antenna arrays. The introduction of the concept of accessible modes, which restricts the analysis to those modes that are observed in a homogeneous region between sharp interfaces, has led to a significant increase in the computational efficiency.
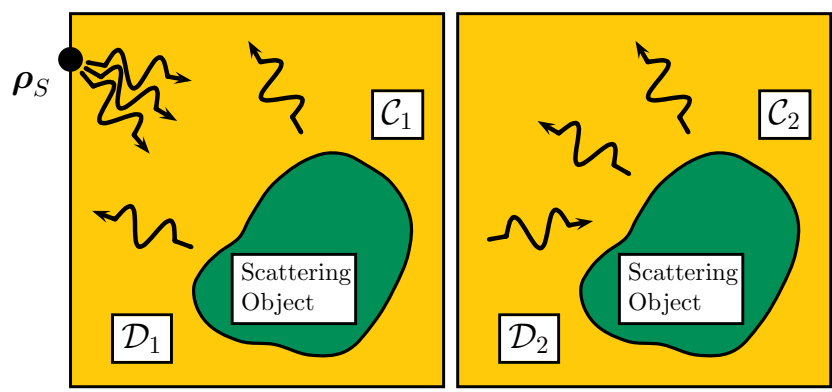

Fig. 1. Excitation of two scattering objects in domains $\mathcal{D}_{1}$ and $\mathcal{D}_{2}$.

In LEGO (Linear Embedding via Green's Operators, [8], [9]), the equivalence principle is used to model the interaction between different subdomains. The advantage is that this method is applicable to boundaries of arbitrary shape. To explain the basic principle, let us consider the two-dimensional multiple-scattering problem shown in Fig. 1. An electrically polarized line source is exciting two identical scattering objects, and the aim is to evaluate the electromagnetic field in an efficient manner. In LEGO, we proceed as follows.

- First, the scattering problem is solved for an isolated object in domain $\mathcal{D}$ with a homogeneous environment in $\overline{\mathcal{D}}$, excited by a line source on the boundary $\mathcal{C}$. The equivalence principle is then used to translate the resulting scattered field in $\overline{\mathcal{D}}$ is into one originating from an equivalent current on $\mathcal{C}$, e.g., by solving an EFIE. This equivalent current distribution is used to define a "scattering operator" for a single object.

- Second, we place a single object in $\mathcal{D}_{1}$, and choose the location of a line source on $\mathcal{C}_{2}$, the boundary of an adjoining domain. With the aid of the known twodimensional Green's function for a homogeneous space, we are then able to translate the "scattering operator" for domain $\mathcal{D}_{1}$ into a "reflection operator" for domain $\mathcal{D}_{2}$.

- Since both domains are identical, we may now combine the scattering and reflection operators for both domains into an integral equation for equivalent currents on $\mathcal{C}_{1}$ and $\mathcal{C}_{2}$. The resulting currents would produce the correct fields inside $\mathcal{D}_{1}$ and $\mathcal{D}_{2}$ in a homogeneous environment. Since the elementary solutions are known from the analysis of a single object, the superposition principle may be invoked to evaluate the field in $\mathcal{D}_{1} \cup \mathcal{D}_{2}$.

The same procedure may be repeated to combine multiple domains. It should be remarked that subdomains need not be identical, and that the order of their combination may be chosen for convenience. This enables us to combine the known portions of a complicated geometry into a fixed environment,

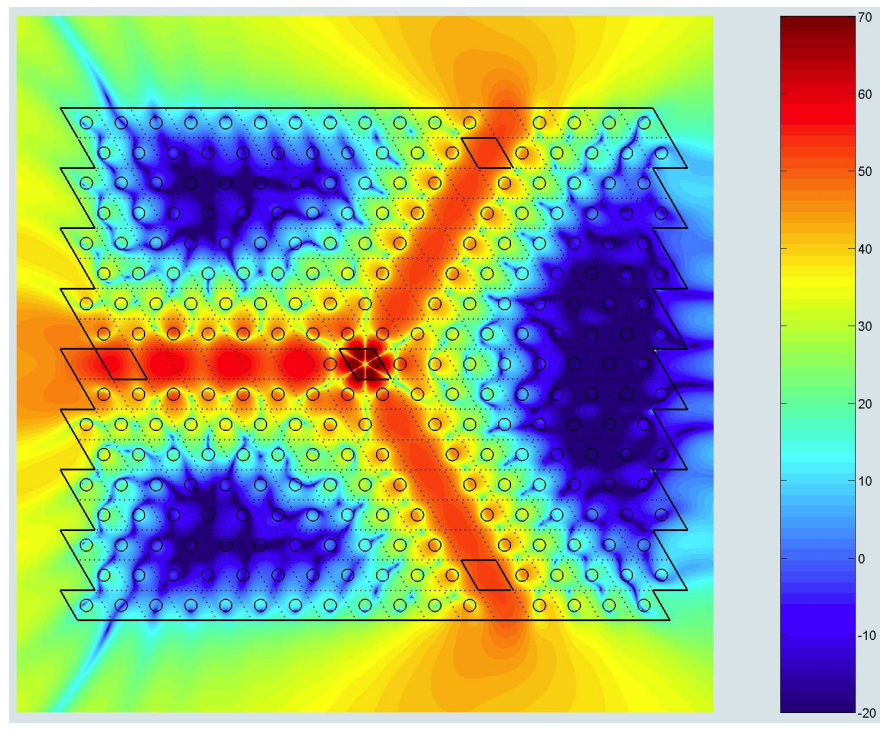

Fig. 2. Electric field in an optimized power splitter (dB scale).

for which a reflection operator is determined for an empty observation domain. By combining this operator with the scattering operator for an object in an empty environment, we obtain the full electromagnetic response. This enables us to optimize an object in the observation domain without reevaluating the response of the complicated environment.

As an illustration, Fig 2 shows the field in an optimized power splitter in a finite EBG structure of $17 \times 17$ cells. In the optimization step, only the radius of a cavity at the junction between the wave-guiding channels was varied. More details can be found in [9].

\section{NONLINEAR OPTIMIZATION}

Like the forward problem, the optimization poses several challenges. We focus on two important aspects.

\section{A. Choice of Cost Function}

Perhaps the most difficult step in the entire procedure is the formulation of the cost function. This is where human intelligence will remain needed. To obtain a first impression of the difficulties that could be encountered, we chose a test structure for which reference data and engineering estimates are available from the literature. We consider an infinite array of rectangular conducting patches on top of a layered dielectric slab and a ground plane, as it is presently being considered for the next generation of phased-array radar systems. To keep the discussion tractable, we approximate the feed by a vertical dipole located below the patch. An impression of a single elementary cell and the relevant physical parameters is given in Fig 3. For the case of a point-source feed, we cannot determine an impedance. Instead, we consider the possibility of generating a circularly polarized wave [10].

Figure 4 shows two objective functions for a varying horizontal position of the source. All other parameters were fixed. 


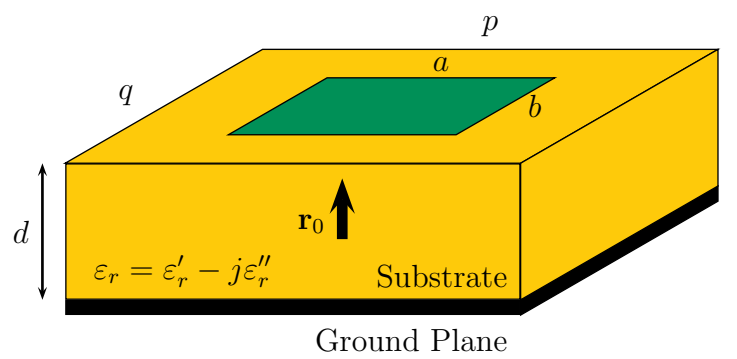

Fig. 3. Element of infinite antenna array and relevant physical parameters.
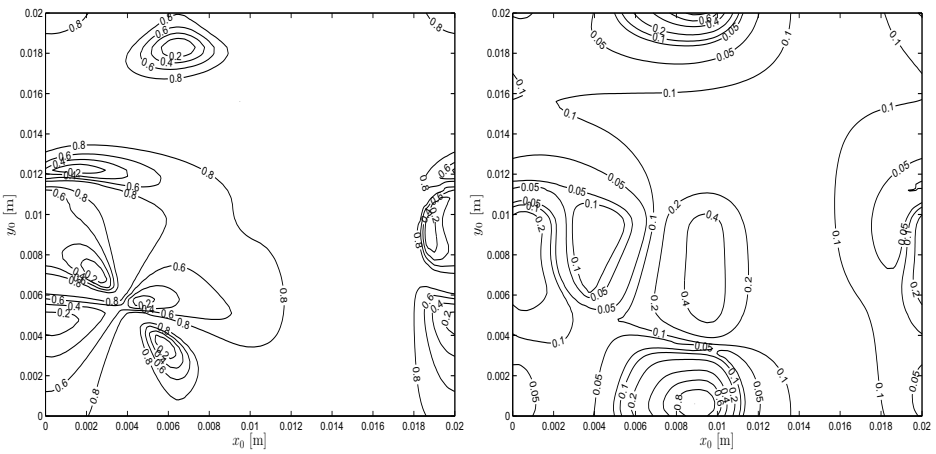

Fig. 4. Objective functions for varying source location

On the left, results are shown for

$$
\operatorname{ERR}=\frac{\left|E_{\theta}^{2}+E_{\varphi}^{2}\right|^{2}}{\left.|| E_{\theta}\right|^{2}+\left.\left|E_{\varphi}\right|^{2}\right|^{2}},
$$

while on the right conditions on the phase and the argument of $E_{\theta}$ and $E_{\varphi}$ were combined into a single cost function. Only for the cost function in (5) convergence was obtained, with the BFGS method. The function defined in (5) equally favors left and right circular polarization. Functions for a single polarization were also investigated, and show less minima.

\section{B. Sensitivity Analysis}

In order to evaluate the sensitivity of the objective function with respect to a physical parameter $p$, we need the derivative $u^{\prime}(p)=\partial_{p} u(p)$. In the context of a parameter sweep, it is tempting to evaluate this derivative by a finite-difference approximation of the form

$$
\left.\partial_{p} u_{p}\right|_{p=p_{m}}=\frac{u\left(p_{m+1}\right)-u\left(p_{m-1}\right)}{2 \Delta p} .
$$

However, when the $\left\{u\left(p_{m}\right)\right\}$ are evaluated by an iterative procedure, they may contain uncorrelated errors, which are enlarged by the division by the step $\Delta p$ in (6). Subtracting $u\left(p_{m-1}\right)$ from $u\left(p_{m+1}\right)$ in the numerator of (6) cancels the leading term in the computed results, so that the error in $u^{\prime}(p)$ may become unnecessarily large.

For a single physical parameter $p$, differentiating (1) yields

$$
L(p) u^{\prime}(p)=f^{\prime}(p)-L^{\prime}(p) u(p),
$$

where the prime indicates a derivative, and where $L^{\prime}(p)$ is available in closed form. By determining $u^{\prime}(p)$ from (7), it is obtained with a similar accuracy as $u(p)$. The evaluation of the additional forcing term $L^{\prime}(p) u(p)$ is usually much faster than the solution of the linear system; moreover, (1) and (7) share the same system matrix.

Now, even the test problem defined in Subsection IV-A already has ten design parameters, i.e., $p, q, a, b, \varepsilon^{\prime} r, \varepsilon_{r}^{\prime \prime}, d$ and the three coordinates in $\mathbf{r}_{0}$. This means that the generation of the search direction in each line-search step from either (6) or (7) takes an extra computational effort equivalent to performing ten field computations. On the other hand, we do not require $u(p)$, but an observable of the form

$$
F(p)=\langle u(p) \mid g(p)\rangle,
$$

where $g(p)$ is a known weighting function. Differentiating (8) with respect to a real-valued parameter $p$ results in

$$
F^{\prime}(p)=\left\langle u^{\prime}(p) \mid g(p)\right\rangle+\left\langle u(p) \mid g^{\prime}(p)\right\rangle .
$$

If we now solve the adjoint problem

$$
L^{\dagger}(p) v(p)=g(p)
$$

we can obtain the derivative of the observable $F(p)$ from

$$
\begin{aligned}
F^{\prime}(p) & =\left\langle u^{\prime}(p) \mid L^{\dagger}(p) v(p)\right\rangle+\left\langle u(p) \mid g^{\prime}(p)\right\rangle \\
& =\left\langle L(p) u^{\prime}(p) \mid v(p)\right\rangle+\left\langle u(p) \mid g^{\prime}(p)\right\rangle \\
& =\left\langle f^{\prime}(p)-L^{\prime}(p) u(p) \mid v(p)\right\rangle+\left\langle u(p) \mid g^{\prime}(p)\right\rangle
\end{aligned}
$$

Thus, the evaluation of all sensitivities or Fréchet derivatives requires only one additional solution of a system of linear equations. During the line search, a single search parameter is varied, and using either (6) or (7) may be more efficient.

\section{REFERENCES}

[1] L. Knockaert and D. de Zutter, "Laguerre-SVD reduced-order modelling”, IEEE Trans. Microwave Theory Tech., vol. 48, pp. 2321-2329, 2004.

[2] P.J. Heres, Robust and Efficient Krylov Subspace Methods for Model Order Reduction, Ph.D. Thesis, Eindhoven, 2005.

[3] A.G. Tijhuis, M.C. van Beurden and A.P.M. Zwamborn, "Iterative solution of field problems with a varying physical parameter", Elektrik, vol. 10, pp. 163-183, 2002.

[4] L. Matekovits, V.A. Laza and G. Vecchi, "Analysis of large complex structures with the synthetic-functions approach", IEEE Trans. Antennas Propagat., vol. 55, pp. 2509-2521, 2007.

[5] V. Prakash and R. Mittra, "Characteristic basis function method: A new technique for efficent solution of method of moments matrix equations", Micr. Opt. Technol. Lett., vol. 36, pp. 95-100, 2003.

[6] D.J. Bekers, S.J.L. van Eijndhoven, A.A.F. van de Ven, P.-P. Borsboom and A.G. Tijhuis, "Eigencurrent analysis of resonant behavior in finite antenna arrays", IEEE Trans. Microwave Theory Tech., vol. 54, pp. 2821-2829, 2006.

[7] S. Monni, G. Gerini, A. Neto and A.G. Tijhuis, "Multimode equivalent networks for the design and analysis of frequency selective surfaces", IEEE Trans. Antennas Propagat., vol. 55, pp. 2824-2835, 2007.

[8] A.M. van de Water, B.P. de Hon, M.C. van Beurden, A.G. Tijhuis and P. de Maagt, "Linear embedding via Green's operators: A modeling technique for finite electromagnetic bandgap structures", Phys. Rev. E., vol. 72 , p. $056704,2005$.

[9] A.M. van de Water, LEGO: Linear Embedding via Green's Operators, $\mathrm{Ph} . \mathrm{D}$. Thesis, Eindhoven, 2007.

[10] R. Dirks, Analysis and Optimization of Large Patch-Antenna Arrays, M.Sc. Thesis, Eindhoven, 2005. 